\title{
Home Automation: HMM based fuzzy rule engine for ambient intelligent smart space
}

\author{
Gopal Singh Jamnal \\ School of Computing \\ Edinburgh Napier University \\ Edinburgh, UK
}

\author{
Xiaodong Liu \\ School of Computing \\ Edinburgh Napier University \\ Edinburgh, UK
}

\author{
Lu Fan \\ Payfont Ltd. \\ Edinburgh, UK
}

\begin{abstract}
-in this paper, we proposed a new type of decisionmaking system to achieve the intelligent goal for automated smart environments. The artificial intelligence techniques, used as building blocks to understand inhabitant activity patterns. The collected information fused to a central inference engine based on Hidden Markov model and Fuzzy rules for taking appropriate actions to communicate and control various home appliances. We proposed a novel CASH (cognitive automated smart home) architecture, based on the Hidden Markov Model and the fuzzy rule based system. The Hidden Markov Model and fuzzy rules are well equipped to address the spatio-temporal activity pattern recognition problem and to trigger appropriate task execution rules.
\end{abstract}

Keywords - home automation; rule mining; smart home; artificial intelligence; expert system; cyber physical system.

\section{INTRODUCTION}

The rapid use of interconnect network objects such as embedded-sensor, RFIDs, BSN are in trend. These interconnected technology known as cognitive Internet of Things (CIoT). Artificial intelligence provides foundation as build blocks to design automated IoT systems. The aim of an ambient intelligent system is to provide comfortable assisted living to inhabitants. Scientific work has been done in ambient intelligent space such as Care-lab, CASAS, Grator-Tech HIS, aware home, iDorm and MavHome projects, the process of activity recognition is subdivided into four part as (i)sensing, (ii)data-preprocessing, (iii)data modelling for feature extraction (iv)feature selection [1]. Many blue-chip companies including IBM Watson, researching into identifying inhabitants preference, activity patterns to provide a customized digital assistant for granting access and controlling various appliance to automated tasks[2].

For recognizing inhabitant activity patterns in smart home scenario, Hidden Markov Model plays a key role. HMM is a generative probabilistic model used for identifying hidden states $<s 1, s 2, . ., s n>$ from given observation sequences $<01,02, . . o n>[3]$. Furthermore, [1] suggested that observable state sequence(st1) at time $t$, depends only on the current state(st), irrespective of previous state (st-1).During HMM training we tend to find optimal state sequence with higher probability of $\operatorname{Pr}(S \mid O)$, known as most likelihood pattern. As an iterative trend, HMM require re-estimation of input parameters to train the system (transition and emission matrix), Baum-welch algorithm work well here as solution. As a result, such re-adjusted parameters increase the probability of finding optimal state sequence. At a later stage, Viterbi algorithm works to find most likelihood patterns.

Furthermore, in later stage, adaptive fuzzy rules have been applied for task executions. The rule based system represents the human expert's knowledge, encoded in set of rules that tell system what to do and what to accomplish in different activity situation as set of if-then statements(if activities(a1, a2 and a3 is active) - then rules(r1,r2 and r3 activated).[4].

\section{CASH (COGNITIVE AUTOMATED SMART HOME) FRAMEWORK}

The proposed CASH architecture, works in two components: (i) recognizing inhabitant activity from sensor's data, (ii) task execution through fuzzy rules activation. In first component, embedded sensors capture activity sequences of inhabitants and identify Hidden likelihood patterns of activities. While in second component, fuzzy rule adaptation applied for task execution, the set of likelihood activity patterns mapped with fuzzy rules. Fuzzy rules invoke operations on home appliances for switching On/Off, set temperature etc.

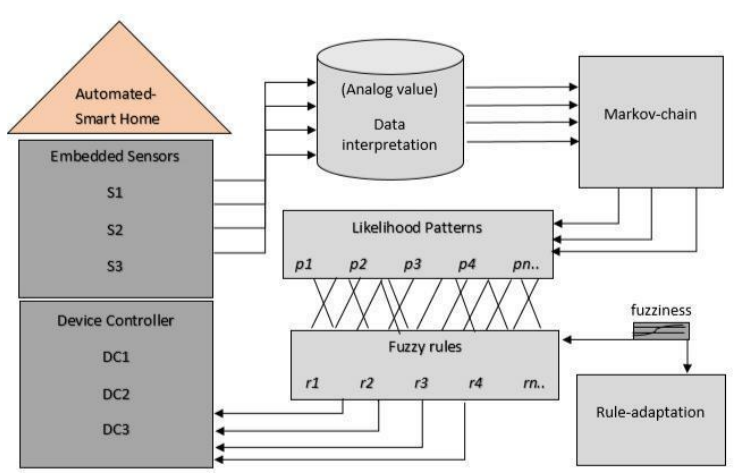

Figure 1. The proposed CASH (cognitive automated smart home). 
In the use case scenario, we placed 9 sensors capturing light, motion, noise inside a studio flat. These sensors are well programmed with Arduino Uno Wi-Fi micro-controller and placed appropriately inside bedroom, living room and kitchen area. Hidden Markov model analyze datasets to identify daily routine activity sequences of inhabitant. The proposed CASH model, trained over captured data sets for multiple time using Balm-welch and Viterbi algorithms to obtain most likelihood pattern of activity sequence. As human activity are overlapped and fuzzy in nature, therefore applying fuzzy rules is right solution to the problem. Fuzzy rules are applied on identified activity pattern to control various appliances inside home for providing a comfortable automated living to inhabitant.

Table 1. Sensor’s digital state for activity labeling

\begin{tabular}{|c|c|c|c|c|c|c|c|}
\hline $\begin{array}{r}\text { time- } \\
\text { sequence }\end{array}$ & $t$ & $t+1$ & $t+2$ & $t+3$ & $t+4$ & $t+5$ & $t+6$ \\
\hline activity & & & & & & & \\
\hline reading & 0 & 0 & 1 & 0 & 0 & 1 & 0 \\
\hline Woking & 1 & 1 & 1 & 1 & 1 & 1 & 1 \\
\hline cooking & 0 & 1 & 0 & 0 & 0 & 0 & 1 \\
\hline Sleeping & 0 & 0 & 0 & 0 & 0 & 0 & 1 \\
\hline
\end{tabular}

The nine sensors, capturing motion $(m)$, light $(l)$ and noise (n), have given unique ID for data interpretation. The motion sensors, track inhabitant mobility so placed in center and light sensor placed in the corner area while sound sensor placed near by TV set. All sensors are connected to Arduino UNO Wi-Fi microcontroller as access point.

In CASH framework, fuzzy rules linked up with activity patterns as < antecedent $>$ and $<$ consequent $>$ manner. In table 2 , various rules defined to automate appliances in smart home for providing a comfort living for inhabitants. Those fuzzy rules provide $<$ consequent $>$ interface for inference engine such as $I F$ (likelihood pattern $p 1, p 2, p 3$ identified) THEN trigger rules (R1, R2, R3).

Table 2. Automated tasks as fuzzy rules.

\begin{tabular}{|l|l|l|}
\hline ID & \multicolumn{1}{|c|}{$\begin{array}{c}\text { Fuzzy } \\
\text { rule }\end{array}$} & \multicolumn{1}{|c|}{ Automated task for activity } \\
\hline 1 & R1 & $\begin{array}{l}\text { Tea kettle on } \rightarrow \text { lock doors } \rightarrow \text { set temp. } \\
\rightarrow \text { switch off TV } \rightarrow \text { switch on study } \\
\text { lamp }\end{array}$ \\
\hline 2 & R2 & $\begin{array}{l}\text { Switch on TV favorite channel, set } \\
\text { temp. } \rightarrow \text { dim light }\end{array}$ \\
\hline 3 & R3 & $\begin{array}{l}\text { Warm up oven } \rightarrow \text { tea kettle ON } \rightarrow \text { ask } \\
\text { for laundry/dishwashing }\end{array}$ \\
\hline 4 & R4 & $\begin{array}{l}\text { Lock doors } \rightarrow \text { set temp } \rightarrow \text { control blinds } \\
\rightarrow \text { switch off TV }\end{array}$ \\
\hline 5 & R5 & $\begin{array}{l}\text { Set temp } \rightarrow \text { tea kettle on } \rightarrow \text { study lamp } \\
\text { ON }\end{array}$ \\
\hline
\end{tabular}

We used 250 data sets of observed sequence over three days from 01/01/2017 to 03/01/2017, which are labelled based on each activity type. The training set and testing set have been divided into 180 and 70 sets respectively. Matlab 2016 version were used for experiments, furthermore 180 observed activity datasets have been labelled into four main activities. Initial transition and emission matrix have been defined based on prior knowledge base. Afterwards, training and testing performed using Matlab's hmmestimate and hmmtrain methods, Baum-welch algorithm applied for further re-estimation of transition and emission probability matrix parameters and train system with new parameters. Matlab experiments trained the system over 180 observed sequences. Finally, hmmviterbi methods used to apply Viterbi algorithm to identify most likelihood activity sequences. Using 80 testing activity datasets, system achieve $79 \%$ of accuracy, shown in fig. 2. as Matlab simulation result.

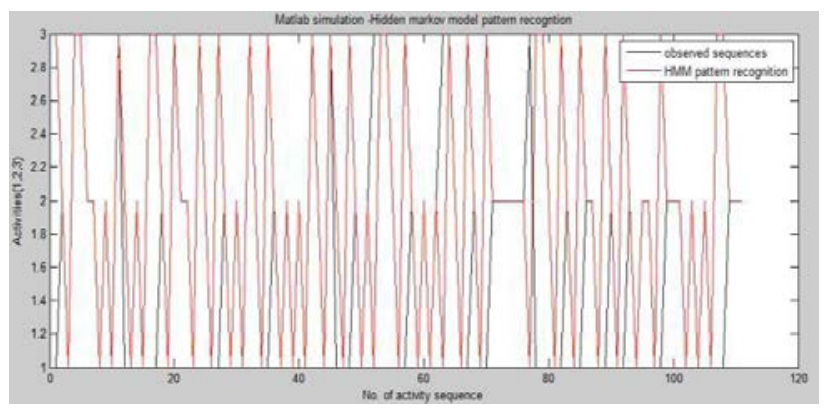

Fig. 2. HMM likelihood activity sequence compares to observedsequence.

\section{CONCLUSION}

This paper proposed a new type of CASH (Cognitive Automated Smart Home) architecture to provide comfortable inhabitant daily living by understanding their needs and activity intention. We embraced the knowledge base from traditional context aware pervasive computing and combined with the Cognitive Internet of things. Furthermore, the building block of CASH architecture is well equipped with artificial intelligence machine learning algorithms of Hidden Markov Model and fuzzy rules-based system. CASH recognize hidden pattern in daily activity living and apply appropriate rule for task execution. Moreover, CASH framework, can be applied in elderly health care system, smart classrooms, and smart spaces for automated environment

\section{REFERENCES}

G. Jamnal and X. Liu, "A cognitive-IoE approach to ambientintelligent smart home,” IoTBDS 2017., in press.

Ibm.com, 'IBM Watson Internet of

Things(IoT)', [2017]. [Online]. Available:

https://www.ibm.com/internet-of-things/.

[Accessed: 01-Apr-2017].

[3] M. R. Hassan, K. Ramamohanarao, J. Kamruzzaman, M. Rahman, and M. Maruf Hossain, "A HMM-based adaptive fuzzy inference system for stock market forecasting,” Neurocomputing, vol. 104, no. July 2016, pp. 10-25, 2013. Syst., vol. 17, pp. 149-185. 